\title{
Baro-excited neurons in the caudal ventrolateral medulla (CVLM) recorded using the whole-cell patch-clamp technique
}

\author{
Naoki Oshima ${ }^{1,2}$, Hiroo Kumagai ${ }^{1}$, Kamon Iigaya ${ }^{2}$, Hiroshi Onimaru ${ }^{3}$, Akira Kawai $^{3}$, Yasuhiro Nishida ${ }^{4}$, \\ Takao Saruta ${ }^{2}$ and Hiroshi Itoh ${ }^{2}$
}

Caudal ventrolateral medulla (CVLM) neurons have important roles in the regulation of sympathetic nerve activity and blood pressure through their tonic inhibition of rostral ventrolateral medulla neurons. As few reports have demonstrated CVLM neuronal activity using the whole-cell patch-clamp technique, we attempted to find neurons in the CVLM that are depolarized by the stimulation of baroreceptors. To record the membrane potentials of the neurons in the CVLM, we developed a modified brainstem-spinal cord preparation that enabled us to change the pressure exerted on the aortic arch and carotid sinuses. We were able to identify neurons in the CVLM in which they were depolarized and the action potential (AP) frequency was increased upon baroreceptor stimulation. We referred to these neurons as baro-excited CVLM neurons. When these preparations were superfused with an angiotensin-II (Ang-II) solution, the frequency of the APs increased in 10 of the 14 baro-excited CVLM neurons. Superfusion with a low-Ca ${ }^{2+}$, high- $\mathrm{Mg}^{2+}$ solution abolished the APs in all seven baro-excited CVLM neurons, suggesting that the baro-excited CVLM neurons did not fire spontaneously. When the preparation was superfused with a low- $\mathrm{Ca}^{2+}$ solution, 6 of the 7 baro-excited CVLM neurons did not respond to Ang-II superfusion. We for the first time found the baro-excited CVLM neurons, which depolarized pressure dependently but may not fire spontaneously. As Ang-II did not change the activity of the CVLM neurons during superfusion with a low- $\mathrm{Ca}^{2+}$, high- $\mathrm{Mg}^{2+}$ solution, the presynaptic neurons may be mandatory for the Ang-II-induced activation of postsynaptic baro-excited CVLM neurons.

Hypertension Research (2012) 35, 500-506; doi:10.1038/hr.2011.211; published online 8 December 2011

Keywords: angiotensin-II; baro-excited neurons; CVLM neurons; modified brainstem-spinal cord preparation; whole-cell patch-clamp technique

\section{INTRODUCTION}

The caudal ventrolateral medulla (CVLM) is a critical site for tonic and reflex control of the sympathetic nervous system. ${ }^{1,2}$ However, the activity of CVLM neurons has seldom been investigated because of the technical difficulty in making intracellular recordings of these neurons. Some studies have investigated neuronal activity in the CVLM using extracellular recordings. ${ }^{3-6}$ Schreihofer and Guyenet ${ }^{4,5}$ showed the presence of baro-excited GABAergic neurons in the CVLM region. They also showed that some baro-activated CVLM neurons received glutamatergic inputs from central respiratory neurons and demonstrated that the response of the baro-activated CVLM neurons to hypoxia was related to the basal pattern of respiratory-related activity. ${ }^{6}$ However, as drug sensitivity, membrane potentials and firing patterns of CVLM neurons are fully unknown, we recorded the activities of baro-excited CVLM neurons using the patch-clamp technique to examine their characteristics.
A brainstem-spinal cord preparation is useful in that it preserves the sympathetic neuronal network. ${ }^{7-14}$ In an earlier study, ${ }^{9}$ we performed whole-cell recordings of rostral ventrolateral medulla (RVLM) neurons and classified them into three types according to their discharge patterns. We have also demonstrated monosynaptic connections between neurons in the RVLM and sympathetic pre-ganglionic neurons by simultaneous recording using the same preparation. ${ }^{13}$ Furthermore, we identified a monosynaptic (axonal) projection extending from the caudal end of the ventrolateral medulla to the inter-mediolateral cell column using optical imaging and recorded the electrical transmission using a motion picture. ${ }^{12}$

In the present study, we developed a modified brainstem-spinal cord preparation in which the aortic arch, carotid sinuses and baroreceptor afferent nerves remained intact. This new preparation enabled us to record the membrane potentials of baro-sensitive neurons in the brainstem and spinal cord region. Using this preparation

${ }^{1}$ Department of Nephrology, National Defense Medical College, Saitama, Japan; ${ }^{2}$ Department of Internal Medicine, Keio University School of Medicine, Tokyo, Japan; ${ }^{3}$ Department of Physiology, Showa University School of Medicine, Tokyo, Japan and ${ }^{4}$ Department of Physiology, National Defense Medical College, Saitama, Japan 
we recorded the membrane potentials of CVLM neurons using the patch-clamp technique. In an earlier study, ${ }^{10}$ we showed that an angiotensin-II (Ang-II) superfusion depolarized the membrane potential and increased the firing of the RVLM neurons, whereas an Ang-II-receptor blocker, candesartan, hyperpolarized the RVLM neurons. Furthermore, previous studies indicated the existence of $\mathrm{AT}_{1}$ receptors in the RVLM. ${ }^{15,16}$ Ang-II is considered to have an influence on RVLM neurons. So, we examined whether Ang-II may have some effects on the baro-excited neurons in the CVLM, which are considered to be crucial neurons to send inhibitory signals to RVLM neurons.

In the present study, we superfused the preparation with Ang-II dissolved in the low- $\mathrm{Ca}^{2+}$, high- $\mathrm{Mg}^{2+}$ solution to determine whether Ang-II stimulated the baro-excited CVLM neurons directly.

\section{METHODS}

\section{General preparations}

The experiments were performed using brainstem-spinal cord preparations collected from 1- to 4-day-old Wistar rats, as described previously. ${ }^{7-14,17,18}$ The protocols of the experiments were approved by the Institutional Review Board of Keio University School of Medicine, Tokyo, and were in accord with the National Guidelines for the Conduct of Animal Experiments. With the animals under deep ether anesthesia, the brainstem-spinal cord was isolated together with other organs (including the heart, carotid sinuses and aorta), which were maintained intact (Figure 1). The ventral surface of the brainstem and the dorsal surface of the brainstem-spinal cord were exposed. All the spinal roots were cut to eliminate respiratory movements. The brainstem was sectioned between the roots of cranial nerve VI and the lower border of the trapezoid body. The roots of cranial nerves IX and X, the carotid sinuses, and the aortic arch were maintained intact. The preparation was continuously superfused with a solution containing (in mmoll ${ }^{-1}$ ) $124 \mathrm{NaCl}, 5.0 \mathrm{KCl}, 1.2 \mathrm{KH}_{2} \mathrm{PO}_{4}, 2.4$ $\mathrm{CaCl}_{2}, 1.3 \mathrm{MgCl}_{2}, 26 \mathrm{NaHCO}_{3}$ and 30 glucose, and maintained at $25-26^{\circ} \mathrm{C}$ (artificial cerebrospinal fluid (CSF)). The $\mathrm{pH}$ (7.4) and oxygenation of the solution were maintained by bubbling the solution with $90 \% \mathrm{O}_{2}-5 \%$ $\mathrm{N}_{2}-5 \% \mathrm{CO}_{2}$.

A catheter (intravenous catheter for cut-down, outer diameter, $1.0 \mathrm{~mm}$, polyethylene; Atom, Tokyo, Japan) was inserted into the descending aorta and used for infusion of artificial CSF; the other end of the catheter was attached to a mercury sphygmomanometer (model 605P; Yamasu, Tokyo, Japan). The tip of the catheter in the descending aorta was thinned (outer diameter, $0.5 \mathrm{~mm}$ ) to fit the diameter of the descending aorta. Various levels of pressure were applied to the aortic arch and carotid sinus by altering the flow of artificial CSF through the catheter inserted into the descending aorta (Figure 1). The flow of artificial CSF was controlled using the inflation bulb and the control valve of the sphygmomanometer. During the experiments, a steady target pressure (such as 60 and $130 \mathrm{~mm} \mathrm{Hg}$ ) was maintained using the sphygmomanometer. Artificial CSF was infused into the catheter through a three-way controller.

\section{Patch-clamp electrodes}

Electrodes were pulled from thin-wall borosilicate filament capillaries (GC100TF-10, outer diameter, $1.0 \mathrm{~mm}$; Clark Electromed, Reading, UK) in one stage using the vertical puller. The electrodes had a tip diameter of 1.8$2.0 \mu \mathrm{m}$ and a resistance of $4-8 \mathrm{M} \Omega$. The electrode solution for whole-cell recording consisted of (in mmoll $\mathrm{l}^{-1}$ ) 130 potassium gluconate, 10 HEPES, 10 EGTA, $1 \mathrm{CaCl}_{2}$ and $1 \mathrm{MgCl}_{2}$ (pH 7.2-7.3, adjusted with $\mathrm{KOH}$ ). The electrode tips were filled with $1 \%$ Lucifer-Yellow (Aldrich Chemical, Milwaukee, WI, USA).

\section{Recording procedure}

A patch-clamp amplifier (AxoPatch, ID; Axon Instruments, Sunnyvale, CA, USA) was used to record the membrane potentials. The CVLM neurons were sought from the ventral side of the medulla. Before making intracellular wholecell recordings, we observed the firing pattern of the target neurons using extracellular recordings. The membrane potentials were recorded using the

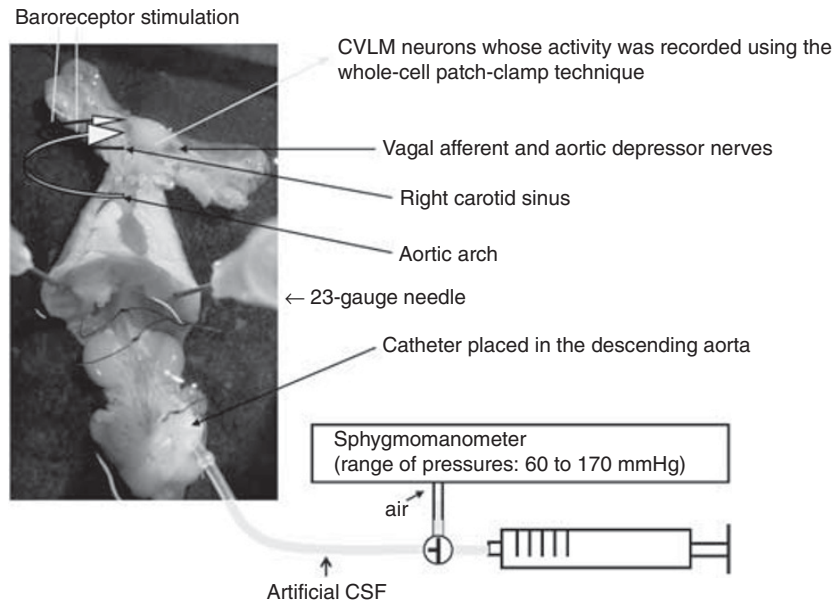

Figure 1 An actual photograph and the scheme of the modified brainstemspinal cord preparation that we have developed. In this preparation, the heart, lung and kidneys were left intact; the aortic arch and carotid sinuses remain in their normal anatomical positions; and the baroreceptor afferent nerves and the aortic depressor nerve are intact. After the start of whole-cell patch-clamp recording of the membrane potentials of the CVLM or RVLM neurons, we stimulated the baroreceptors by increasing the aortic pressure from 60 to $200 \mathrm{~mm} \mathrm{Hg}$ by infusing artificial CSF through a catheter, the tip of which was positioned in the descending aorta. CVLM, caudal ventrolateral medulla; RVLM, rostral ventrolateral medulla. A full color version of this figure is available at the Hypertension Research journal online.

current-clamp technique (20-pA increments from -100 to $20 \mathrm{pA}, 500 \mathrm{~ms}$ in duration) and were corrected for junction potentials at the pipette tip $(-11 \mathrm{mV})$. All the data were recorded and analyzed using PowerLab (AD Instruments, Colorado Springs, CO, USA). During the course of the whole-cell recordings, the neurons were labeled with Lucifer-Yellow either by spontaneous diffusion or iontophoresis. A chloride ion equilibrium potential of $-89 \mathrm{mV}$ was calculated using the Nernst equation, and the intracellular and extracellular chloride concentrations.

\section{Methods}

The aortic arch and carotid sinuses were maintained in their normal anatomical relationships in this preparation, and the aortic depressor nerve and baroreceptor afferent nerves were intact. To determine whether the recorded neurons were baroreceptor-sensitive, we stimulated the baroreceptors by increasing the pressure on the aorta from 60 to $170 \mathrm{~mm} \mathrm{Hg}$ by infusing artificial CSF through the catheter placed in the descending aorta.

\section{Experimental protocols}

Protocol-1. We used the whole-cell patch-clamp technique to record membrane potentials of the CVLM neurons. We examined the responses of the neurons in the CVLM to changes in pressure (ranging from 60 to $170 \mathrm{~mm} \mathrm{Hg}$ ) applied to the aortic arch and carotid sinus so as to simulate an elevation of blood pressure. While recording the activities of the neurons, the pressures on the baroreceptors were generally kept at $60 \mathrm{~mm} \mathrm{Hg}$. We tried to identify baroexcited CVLM neurons based on the following criteria: a whole-cell patchclamp recording showing depolarization of the membrane potential of a CVLM neuron (more than $2 \mathrm{mV}$ in amplitude) when the pressure loaded on the aortic arch and carotid sinuses was increased from 60 to $130 \mathrm{~mm} \mathrm{Hg}$.

Protocol-2. After starting to record the membrane potential of a baro-excited CVLM neuron, the preparation was superfused with $6 \mu \mathrm{moll}^{-1}$ Ang-II (human; Sigma, St Louis, MO, USA) dissolved in artificial CSF, and responses of neurons in the CVLM to the Ang-II superfusion were recorded. The duration of Ang-II superfusion was from 8 to $12 \mathrm{~min}$. During superfusion with Ang-II, we defined depolarization as increase of more than $2 \mathrm{mV}$ in the membrane potential and hyper-polarization as decrease of less than $-2 \mathrm{mV}$ in the 
membrane potential. Change in membrane potentials and action potential frequencies were calculated after 6 to 12-min superfusion with Ang-II.

Protocol-3. After starting to record the membrane potential of a baro-excited CVLM neuron, the preparation was superfused with a low- $\mathrm{Ca}^{2+}$, high- $\mathrm{Mg}^{2+}$ solution ((in mmoll l-1) $124 \mathrm{NaCl}, 5.0 \mathrm{KCl}, 1.2 \mathrm{KH}_{2} \mathrm{PO}_{4}, 0.2 \mathrm{CaCl}_{2}, 5.0 \mathrm{MgCl}_{2}$, $26 \mathrm{NaHCO}_{3}$ and 30 glucose) to block synaptic transmission, ${ }^{19}$ and the responses of the membrane potentials of the baro-excited CVLM neurons to changes in pressure applied to the aortic arch and carotid sinus were examined.

Protocol-4. After superfusion with the low- $\mathrm{Ca}^{2+}$, high- $\mathrm{Mg}^{2+}$ solution for over $30 \mathrm{~min}$, the preparation was superfused with Ang-II $\left(6 \mu \mathrm{moll}^{-1}\right)$ dissolved in the low- $\mathrm{Ca}^{2+}$, high- $\mathrm{Mg}^{2+}$ solution for over $10 \mathrm{~min}$, and the membrane potential of the baro-excited CVLM neuron was examined. The change in membrane potentials was calculated after 30-min superfusion with the low- $\mathrm{Ca}^{2+}$, high$\mathrm{Mg}^{2+}$ solution and 10-min superfusion with Ang-II dissolved in the low-Ca ${ }^{2+}$, high- $\mathrm{Mg}^{2+}$ solution. The definitions of depolarization and hyper-polarization, which were described in Protocol-2, were applied.

\section{Statistics}

Results were expressed as means \pm s.e.m. Comparisons of membrane potentials under pressure of 130 and $170 \mathrm{~mm} \mathrm{Hg}$ were made by Student's $t$-test for paired observation. Statistical significance was set at $P<0.05$.

\section{Histological examination}

At the conclusion of the experiments, the preparations were fixed for at least $48 \mathrm{~h}$ at $4{ }^{\circ} \mathrm{C}$ in phosphate-buffered $10 \%$ formalin and a cryostat was used to cut them into $100-\mu \mathrm{m}$ transverse frozen sections. Lucifer-Yellow-labeled baroexcited CVLM neurons were photographed using a fluorescence microscope (Olympus, Tokyo, Japan). Selected neurons were traced using a camera lucida attachment at a magnification of $\times 400$. Neutral-Red staining was performed to verify the position of the neurons within the CVLM, and the precise location of the marked neurons was identified.

\section{RESULTS}

\section{Baro-excited CVLM neurons}

We succeeded in recording the membrane potentials of the neurons in the CVLM using the whole-cell patch-clamp technique and the modified brainstem-spinal cord preparations. When we increased the aortic pressure from 60 to $130 \mathrm{~mm} \mathrm{Hg}$, the membrane potentials of neurons in the CVLM became depolarized (Figure 2). We referred to these neurons as 'baro-excited CVLM neurons'. We recorded the baro-excited CVLM neurons using the whole-cell patch-clamp technique $(n=21)$, and all the neurons showed irregular firings. ${ }^{9}$ The mean resting membrane potential was $-54.0 \pm 0.9 \mathrm{mV}$, the mean frequency of the action potential (AP) was $0.9 \pm 0.3 \mathrm{~Hz}$ and the input resistance was $895 \pm 55 \mathrm{M} \Omega$.

As shown in Figure 3a, we recorded the responses of the baroexcited CVLM neurons to two different levels of pressure applied to the baroreceptors. We changed the pressure applied to the carotid sinus and aortic arch from 60 to $130 \mathrm{~mm} \mathrm{Hg}$ and to $170 \mathrm{~mm} \mathrm{Hg}$. The whole-cell recordings showed that, the higher the pressure on the baroreceptors, the more depolarized the membrane potentials in the baro-excited CVLM neurons. Eight baro-excited CVLM neurons were tested under pressure from 60 to $130 \mathrm{~mm} \mathrm{Hg}$ and to $170 \mathrm{~mm} \mathrm{Hg}$, and the same reactions were observed in all the neurons. The degree of depolarization to $170 \mathrm{~mm} \mathrm{Hg}$ was significantly larger than that to $130 \mathrm{~mm} \mathrm{Hg}$ (Figure 3b). Therefore, the membrane potentials in the baro-excited CVLM neurons seem to depend on the pressure applied to the baroreceptors.

\section{Effect of Ang-II on baro-excited CVLM neurons}

We examined the effect of Ang-II on 14 baro-excited CVLM neurons recorded using the whole-cell patch-clamp technique. When the

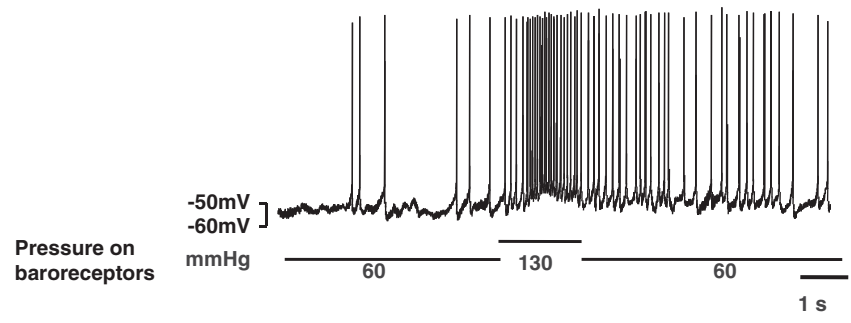

Figure 2 Membrane potentials of a baro-excited neuron in the CVLM. Membrane potentials recorded using the whole-cell patch-clamp technique. When we increased the pressure in the aortic arch and carotid sinuses from 60 to $130 \mathrm{~mm} \mathrm{Hg}$, the membrane potential of the neurons in the CVLM became depolarized and the frequency of the action potentials increased. These neurons were termed baro-excited CVLM neurons. CVLM, caudal ventrolateral medulla.

a

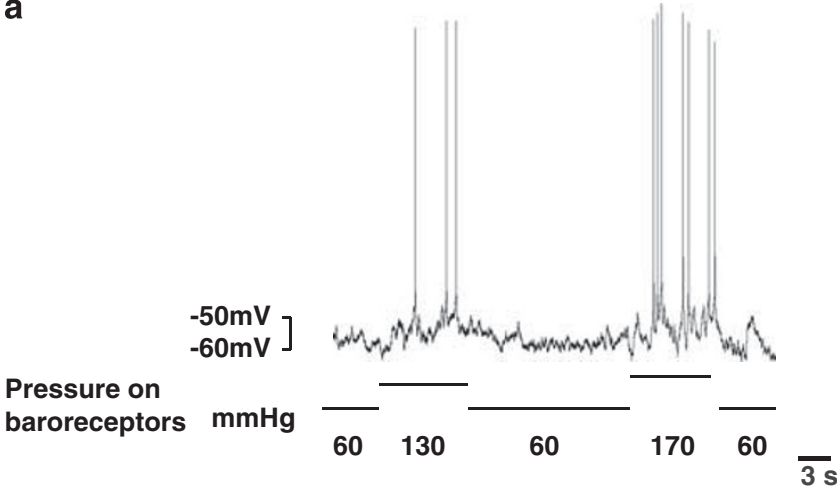

b $\mathrm{mV}$

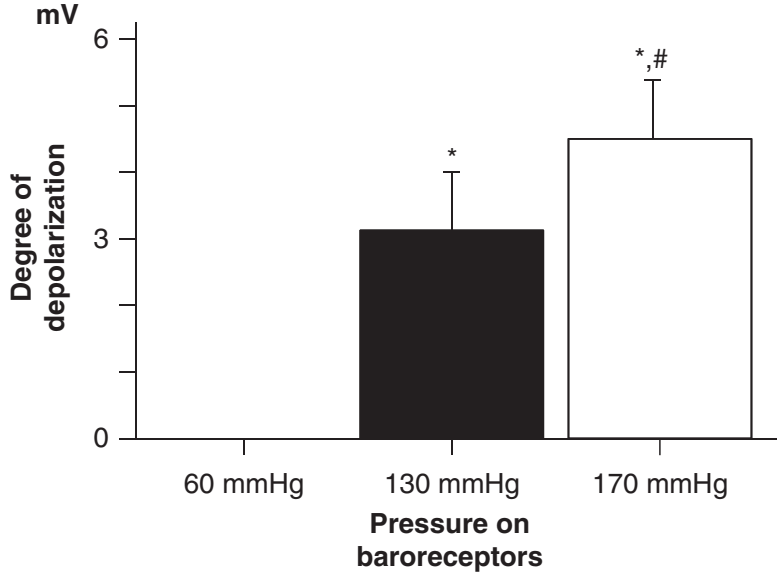

Figure 3 (a) Responses of the baro-excited neuron in the CVLM to three levels of pressure applied on the baroreceptors. Membrane potential was recorded by whole-cell recording. The pressure applied to the carotid sinus and aortic arch was changed from 60 to $130 \mathrm{~mm} \mathrm{Hg}$ and to $170 \mathrm{~mm} \mathrm{Hg}$. This recording shows that the higher the pressure on the baroreceptors, the more depolarized the membrane potential in the baro-excited CVLM neuron. (b) Graphical representation of changes of membrane potentials in baroexcited CVLM neurons $(n=8)$ under pressure applied to the carotid sinus and aortic arch ranging from 60 to $130 \mathrm{~mm} \mathrm{Hg}$ and to $170 \mathrm{~mm} \mathrm{Hg}$. The changes of membrane potentials under the pressure of 130 and $170 \mathrm{~mm} \mathrm{Hg}$ are presented as the degree of depolarization from the membrane potentials under the pressure of $60 \mathrm{~mm} \mathrm{Hg}$. Values are mean \pm s.e.m. ${ }^{*} P<0.001 \mathrm{vs}$. $60 \mathrm{~mm} \mathrm{Hg} ; \quad \#<0.05$ vs. $130 \mathrm{~mm} \mathrm{Hg}$ (Student's $t$-test). CVLM, caudal ventrolateral medulla. 
a

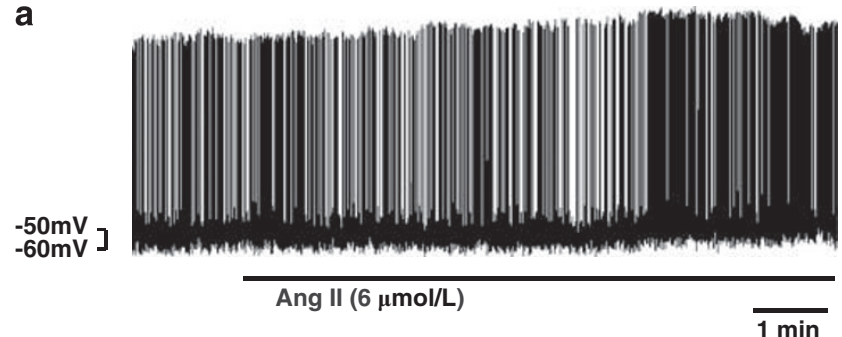

b

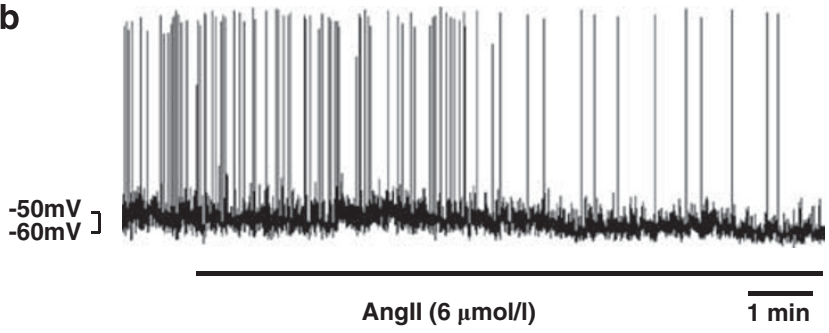

Figure 4 Effects of Ang-II superfusion on baro-excited CVLM neurons during superfusion with artificial CSF. (a) When a preparation was superfused with Ang-II, the membrane potential clearly became depolarized and the frequency of the APs increased. (b) When another preparation was superfused with Ang-II, the membrane potential became hyper-polarized and the frequency of the APs decreased. During these recordings, the pressure on the baroreceptors was maintained at $60 \mathrm{~mm} \mathrm{Hg}$. Ang-II, angiotensin-II; AP, action potential; CSF, cerebrospinal fluid; CVLM, caudal ventrolateral medulla.

Table 1 Effects of Ang-II superfusion on baro-excited CVLM neurons $(n=14)$

\begin{tabular}{lcc}
\hline & $\begin{array}{c}\text { Change in } \\
\text { membrane } \\
\text { potential }\end{array}$ & $\begin{array}{c}\text { Change in frequency } \\
\text { of action potential }\end{array}$ \\
\hline Increase in neuronal activity $(n=10)$ & $+3.8 \pm 0.4 \mathrm{mV}^{*}$ & $+2.2 \pm 0.1 \mathrm{~Hz}$ \\
Decrease in neuronal activity $(n=4)$ & $-4.5 \pm 1.4 \mathrm{mV}^{\#}$ & $-0.8 \pm 0.1 \mathrm{~Hz}$ \\
\hline
\end{tabular}

Abbreviations: Ang-II, angiotensin-II; CVLM, caudal ventrolateral medulla.

Values are mean \pm s.e.m.

${ }^{*} P<0.01$ vs. before Ang-II superfusion; ${ }^{*} P<0.05$ vs. before Ang-II superfusion.

preparations were superfused with Ang-II dissolved in artificial CSF, the membrane potential of 10 of the 14 baro-excited CVLM neurons clearly depolarized and the frequencies of the APs increased (Figure 4a). As shown in Table 1, the activities of 10 of the 14 neurons increased after Ang-II superfusion, and their membrane potentials were depolarized by $+3.8 \pm 0.4 \mathrm{mV}(P<0.01)$ (Table 1$)$.

By contrast, the membrane potentials of the other four baro-excited CVLM neurons hyperpolarized, and the frequencies of the APs decreased (Figure $4 \mathrm{~b}$ ). The activities of the other four neurons were decreased by Ang-II superfusion, and their membrane potentials were hyperpolarized by $-4.5 \pm 1.4 \mathrm{mV}(P<0.05)$ (Table 1$)$.

Effect of low-Ca ${ }^{2+}$, high-Mg ${ }^{2+}$ superfusion on baro-excited CVLM neurons

After starting to record the membrane potential of the baro-excited CVLM neurons, we superfused the preparations with a low- $\mathrm{Ca}^{2+}$, high- $\mathrm{Mg}^{2+}$ solution to determine whether the baro-excited CVLM neurons fired spontaneously, as a low- $\mathrm{Ca}^{2+}$, high- $\mathrm{Mg}^{2+}$ solution has been shown to block synaptic transmission ${ }^{19}$ from other neurons to

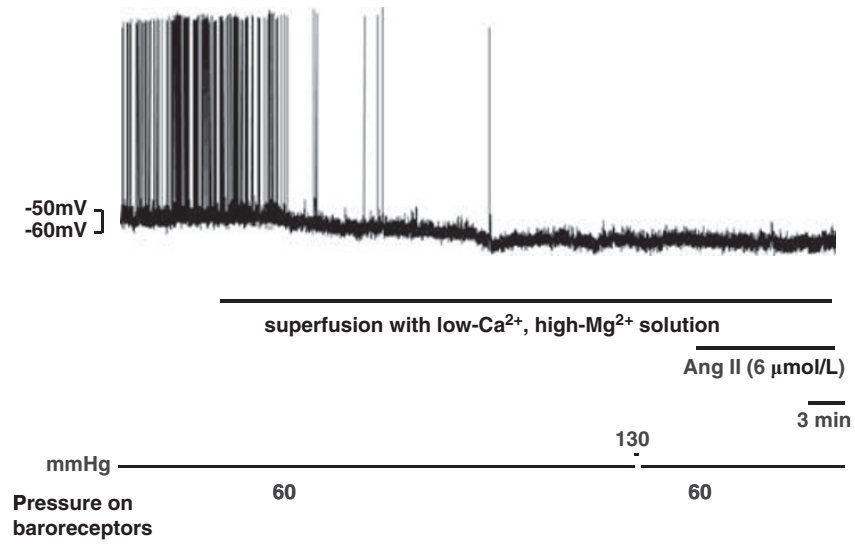

Figure 5 Effects of a low- $\mathrm{Ca}^{2+}$, high- $\mathrm{Mg}^{2+}$ solution on baro-excited CVLM neurons and effects of Ang-II during low-Ca ${ }^{2+}$, high- $\mathrm{Mg}^{2+}$ superfusion. When superfused with the low- $\mathrm{Ca}^{2+}$, high- $\mathrm{Mg}^{2+}$ solution, the membrane potential of the baro-excited CVLM neurons became hyper-polarized and the APs were completely abolished. After superfusion with the low- $\mathrm{Ca}^{2+}$, high- $\mathrm{Mg}^{2+}$ solution for $40 \mathrm{~min}$, the membrane potential did not change when superfused with Ang-Il dissolved in the low- $\mathrm{Ca}^{2+}$, high- $\mathrm{Mg}^{2+}$ solution. During recording of neuron activity, the pressure on the baroreceptors was maintained at $60 \mathrm{~mm} \mathrm{Hg}$. Under superfusion with the low- $\mathrm{Ca}^{2+}$, high- $\mathrm{Mg}^{2+}$ solution, the pressure was changed from 60 to $130 \mathrm{~mm} \mathrm{Hg}$. However no response was observed. Ang-II, angiotensin-II; AP, action potential; CVLM, caudal ventrolateral medulla.

the recorded baro-excited CVLM neurons. We examined the effect of the low- $\mathrm{Ca}^{2+}$, high- $\mathrm{Mg}^{2+}$ solution on seven baro-excited CVLM neurons recorded using the whole-cell patch-clamp technique (Figure 5). The membrane potentials of all seven CVLM neurons became hyperpolarized $(-4.2 \pm 0.4 \mathrm{mV}, P<0.01)$ and their APs were completely abolished by the low- $\mathrm{Ca}^{2+}$, high- $\mathrm{Mg}^{2+}$ solution (Figure 5).

As shown in Figure 5, after $30 \mathrm{~min}$ of superfusion with the low$\mathrm{Ca}^{2+}$, high- $\mathrm{Mg}^{2+}$ solution, the pressure applied to the aortic arch and carotid sinuses was increased, and the response of the membrane potential of the baro-excited CVLM neurons was recorded. None of the seven neurons responded to an increase in the aortic pressure from 60 to $130 \mathrm{~mm} \mathrm{Hg}$ during the low- $\mathrm{Ca}^{2+}$ superfusion.

Effect of Ang-II on baro-excited CVLM neurons during background superfusion with a low- $\mathrm{Ca}^{2+}$, high- $\mathrm{Mg}^{2+}$ solution

After superfusion with the low- $\mathrm{Ca}^{2+}$, high- $\mathrm{Mg}^{2+}$ solution for 30 or $40 \mathrm{~min}$, the preparations were superfused with Ang-II dissolved in the low- $\mathrm{Ca}^{2+}$, high- $\mathrm{Mg}^{2+}$ solution (Figure 5, right end). The membrane potentials of 6 of the 7 neurons remained unchanged $(-0.6 \pm 0.1 \mathrm{mV})$ after Ang-II superfusion. The membrane potential of one neuron depolarized $(+2.1 \mathrm{mV})$ and low-frequency APs were recorded $(0.05 \mathrm{~Hz})$.

\section{Histological localization}

After recordings, we identified the location of the 7 out of 21 recorded CVLM neurons. Lucifer-Yellow staining showed that these seven neurons were located in the CVLM (Figures 6A and B).

\section{DISCUSSION}

Modified brainstem-spinal cord preparation and identification of baro-excited neurons in the CVLM

We identified neurons in the CVLM that depolarized in response to baroreceptor loading and referred to these neurons as 'baro-excited CVLM neurons'. These neurons were thought to suppress the activities 

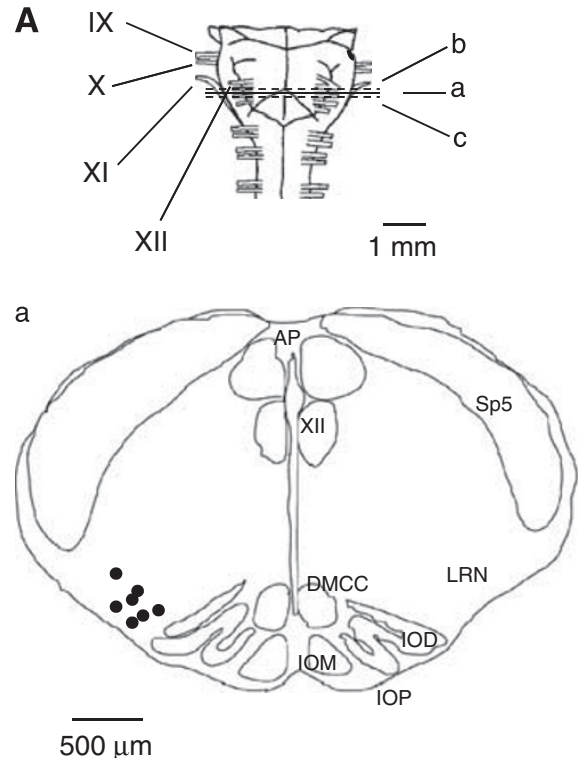

B

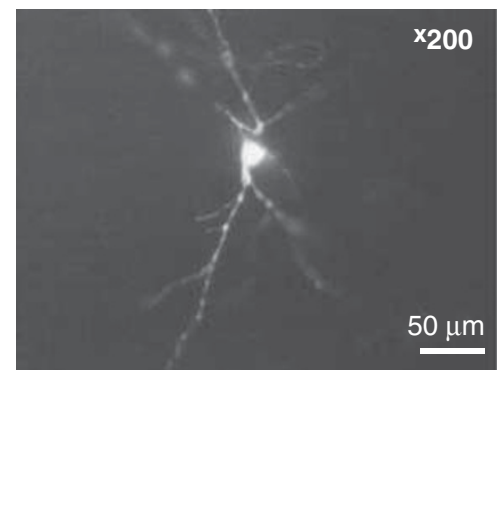

Figure 6 (A) Distribution of the recorded seven baro-excited CVLM neurons. Top: These neurons were recorded within $\pm 200-\mu \mathrm{m}$ distance from the dotted lines (b, c). IX, X and XII represent cranial nerves. Bottom: Cells were plotted on the intermediate cross-section (line a). Sp5, trigeminal spinal tract nucleus; DMCC, dorsomedial cell column; IOM, medial inferior olive; IOP, principle inferior olive; IOD, dorsal inferior olive; LRN, lateral reticular nucleus; XII, hypoglossal nucleus; AP, area postrema. (B) Baro-excited CVLM neurons stained with Lucifer-Yellow. CVLM, caudal ventrolateral medulla. A full color version of this figure is available at the Hypertension Research journal online.

of baro-inhibited RVLM neurons. The higher the pressure applied to the baroreceptors were, the more the neurons in the nucleus tractus solitarius (NTS) depolarized through afferent nerves originated from aortic and carotid baroreceptors, and the more increased signals on those neurons were sent to the baro-excited neurons in the CVLM (Figures $3 \mathrm{a}$ and $\mathrm{b}$ ). This finding suggests that, as systemic blood pressure increases, the activities of the baro-excited CVLM neurons also increase. In spontaneously hypertensive rats, the baroreflex pathway is considered to be disordered. ${ }^{20-22}$ In this study, in Wistar rats the baro-excited neurons in the CVLM depolarized pressure dependently. However, we speculate that those neurons may depolarize less pressure dependently in spontaneously hypertensive rats than in Wistar rats.

This modified brainstem-spinal cord preparation with an intact aortic arch, carotid sinuses and an aortic depressor nerve is useful for studying baro-sensitive neurons in the medulla oblongata, such as neurons in the CVLM and the RVLM. We succeeded in recording baro-excited CVLM neurons using the whole-cell patch-clamp technique in modified brainstem-spinal cord preparations. To our knowledge, no other studies have reported activities of baro-excited CVLM neurons recorded using the whole-cell patch-clamp technique.

Paton ${ }^{23}$ reported a working heart-brainstem preparation in which the baroreflex network also remained intact; they succeeded in performing whole-cell recordings of the neurons in the NTS. The preparation that we have developed is similar to the working heart-brainstem preparation, and the baroreflex pathway from the baroreceptors on the aortic arch and carotid sinuses to the sympathetic pre-ganglionic neurons was maintained intact. This preparation enabled us to record the baro-sensitive activity of the ventral neurons in the brainstem using the patch-clamp technique.

\section{Baro-excited CVLM neurons}

The CVLM region contains GABAergic and cholinergic cardiovagal motor neurons. Extensive studies by Schreihofer and Guyenet ${ }^{4,5}$ have shown that most baro-activated CVLM neurons are GABAergic. Nine of the 12 baro-activated CVLM neurons contained intense hybridization reaction products for GAD67 mRNA, and the other three neurons showed weak hybridization signals for GAD67 mRNA. The CVLM region is reported to also contain neurons that show choline acetyltransferase immunoreactivity. However, none of the baroactivated CVLM neurons that were examined (0/8 neurons) were choline acetyltransferase-immunoreactive. ${ }^{5}$ We did not examine whether the baro-excited CVLM neurons were GABAergic or cholinergic. However, based on the results of the above-mentioned previous studies, we suspect that most of the baro-excited CVLM neurons that we recorded were GABAergic.

Although respiratory inputs to baro-activated CVLM neurons have been reported, ${ }^{6,24}$ we were unable to identify this type of neuron in the present study. Thus, further studies are needed.

\section{Responses of baro-excited CVLM neurons to Ang-II during artificial CSF superfusion}

Membrane depolarization and an increase in the AP frequency were observed by superfusion with Ang-II in 10 of the 14 baro-excited CVLM neurons, whereas hyper-polarization and a decrease in the AP frequency were observed in the four other neurons. These results suggest that Ang-II superfusion appeared to increase the activities of most of the baro-excited CVLM neurons. In our previous study, Matsuura et al. ${ }^{10}$ showed that, during Ang-II superfusion bulbospinal RVLM neurons were more depolarized in spontaneously hypertensive rats than in Wistar-Kyoto (WKY) rats on the brainstem-spinal cord preparations. Some of these results may be induced by the effects exerted by Ang-II on baro-excited CVLM neurons. During Ang-II superfusion the baro-excited neurons in the CVLM may be less depolarized in spontaneously hypertensive rats than in Wistar rats. Further study will be needed.

Sasaki and Dampney ${ }^{25}$ showed that Ang-II microinjected in the RVLM region increased peripheral sympathetic nerve activity, whereas injection of the peptide in the CVLM region decreased the peripheral sympathetic activity. These results indicate that the CVLM neurons, 
which form inhibitory synapses with the RVLM neurons, were activated by Ang-II. Muratani et al. ${ }^{26}$ and Alzamora et al. ${ }^{27}$ obtained similar results. As the baro-excited CVLM neurons formed synapses with the RVLM neurons, these previous results ${ }^{25-27}$ support our present data.

However, the findings of their studies and ours do not answer the question as to whether Ang-II activates the baro-excited CVLM neurons directly. Considering the results of previous studies, ${ }^{25-27}$ we cannot deny the possibility that Ang-II may have activated non-baroexcited CVLM neurons and that these neurons in turn sent synaptic activity to the baro-excited CVLM neurons.

From these studies and our study, the baroreceptor-induced excitation of CVLM neurons may depend on neurons that project to the baro-excited CVLM neurons. Because of the possibility that Ang-II may have activated other neurons (including NTS neurons) with synapses to the baro-excited CVLM neurons in our study, we attempted to examine the direct effect of Ang-II on baro-excited CVLM neurons.

\section{Do baro-excited CVLM neurons fire spontaneously?}

The membrane potentials of all seven baro-excited CVLM neurons that were tested became hyperpolarized and their APs were abolished when the neurons were superfused with a low- $\mathrm{Ca}^{2+}$, high- $\mathrm{Mg}^{2+}$ solution (Figure 5). Superfusion with the low- $\mathrm{Ca}^{2+}$, high- $\mathrm{Mg}^{2+}$ solution completely abolished the baroreceptor-induced excitation of the baro-excited CVLM neurons (Figure 5), suggesting that the presynaptic neurons, such as the neurons in the NTS, that project to the baro-excited CVLM neurons are mandatory for the baroreceptor-induced excitation of CVLM neurons. In an earlier study, ${ }^{9}$ we identified spontaneously firing RVLM neurons using the same wholecell patch-clamp technique in a similar brainstem-spinal cord preparation. We found that almost $30 \%$ of the RVLM neurons maintained regular firing even during superfusion with the low$\mathrm{Ca}^{2+}$, high- $\mathrm{Mg}^{2+}$ solution, whereas $60 \%$ of the RVLM neurons ceased their irregular firing during the low- $\mathrm{Ca}^{2+}$ superfusion. ${ }^{9}$ As none of the seven baro-excited CVLM neurons fired spontaneously in the present study, our results suggest that most baro-excited CVLM neurons do not fire spontaneously. This result means that, if signals are not sent to the baro-excited neurons in the CVLM from other neurons (probably, neurons in the NTS), RVLM neurons may receive little inhibitory signals. This hypothesis suggests that basal blood pressure may be decided mostly by spontaneous activation of RVLM neurons.

Superfusion with Ang-II dissolved in the low- $\mathrm{Ca}^{2+}$, high- $\mathrm{Mg}^{2+}$ solution did not change the activities of 6 of the 7 baro-excited CVLM neurons that were tested (Figure 5, right end), suggesting that most of the baro-excited CVLM neurons are not sensitive to Ang-II by themselves. This result implies that presynaptic neurons are important for the effect of Ang-II on postsynaptic baro-excited CVLM neurons. To our knowledge, no reports have investigated whether baro-excited CVLM neurons themselves are sensitive to Ang-II. In a study by Li and Guyenet, ${ }^{28}$ the RVLM neurons that axonally project into the intermediolateral cell column were sensitive to Ang-II even during low$\mathrm{Ca}^{2+}$, high- $\mathrm{Mg}^{2+}$ superfusion. In this regard, the baro-excited CVLM neurons seemed to be quite different from the RVLM neurons in that the CVLM neurons by themselves were not sensitive to Ang-II. Although one of the seven baro-excited CVLM neurons was sensitive to Ang-II, it was not nearly as sensitive as the RVLM neurons ${ }^{28}$ related to sympathetic nerve activity.

\section{Limitations of the study}

We were unable to measure systemic blood pressure and the actual blood pressures affecting the aortic arch or carotid sinuses. Although we used a sphygmomanometer to change the pressure applied to the aortic arch and carotid sinuses by increasing the pressure of the solution in the catheter placed in the descending aorta, the sphygmomanometer did not record the actual blood pressure applied to the aortic arch and carotid sinuses. Furthermore, the blood pressure stimulus that was applied to the baroreceptors was static, not pulsatile. A previous study ${ }^{29}$ has demonstrated that a pulsatile pressure caused a greater inhibition of the sympathetic nerve activity than a static pressure. So, the effects of baroreceptor stimulation in this study may be smaller than those that occur in vivo.

\section{Conclusions}

We identified baro-excited neurons in the CVLM using the whole-cell patch-clamp technique. By recording the membrane potentials of those neurons with this method, we could find the following new results. The baro-excited CVLM neurons depolarized pressure dependently, none of them fired spontaneously and most of them did not respond to Ang-II by themselves. These results appear to show the characteristic features of baro-excited CVLM neurons. The characteristics of baro-excited CVLM neurons seem to be quite different from those of RVLM neurons, which are related to sympathetic nerve activity. The modified brainstemspinal cord preparation that we have developed is very useful for assessing the baro-sensitivity of the neurons in the medulla oblongata, which has important roles in the sympathetic nervous system.

\section{CONFLICT OF INTEREST}

The authors declare no conflict of interest.

\section{ACKNOWLEDGEMENTS}

Source of funding: This work was supported by Grants-in-Aid for Scientific Research from the Ministry of Education, Science and Culture of Japan.

1 Pilowsky PM, Goodchild AK. Baroreceptor reflex pathways and neurotransmitters: 10 years on. J Hypertens 2002; 20: 1675-1688.

2 Sved AF, Ito S, Madden CJ. Baroreflex dependent and independent roles of the caudal ventrolateral medulla in cardiovascular regulation. Brain Res Bull 2000; 15: 129-133.

3 Terui N, Masuda N, Saeki Y, Kumada M. Activity of barosensitive neurons in the caudal ventrolateral medulla that send axonal projections to the rostral ventrolateral medulla in rabbits. Neurosci Lett 1990; 16: 211-214.

4 Schreihofer AM, Guyenet PG. The baroreflex and beyond: control of sympathetic vasomotor tone by GABAergic neurons in the ventrolateral medulla. Clin Exp Pharmacol Physiol 2002; 29: 514-521.

5 Schreihofer AM, Guyenet PG. Baro-activated neurons with pulse-modulated activity in the rat caudal ventrolateral medulla express GAD67 mRNA. J Neurophysiol 2003; 89: 1265-1277.

6 Mandel DA, Schreihofer AM. Glutamatergic inputs to the CVLM independent of the NTS promote tonic inhibition of sympathetic vasomotor tone in rats. Am J Physiol Heart Circ Physiol 2008; 295: H1772-H1779.

7 Deuchars SA, Morrison SF, Gilbey MP. Medually-evoked EPSPs in neonatal rat sympathetic preganglionic neurons in vitro. J Physiol 1995; 487: 453-463.

8 Lin HH, Wu SY, Lai CC, Dun NJ. GABA- and glycine-mediated inhibitory postsynaptic potentials in neonatal rat rostral ventrolateral medulla neurons in vitro. Neuroscience 1998; 82: 429-442.

9 Oshima N, Kumagai H, Kawai A, Sakata K, Matsuura T, Saruta T. Three types of putative presympathetic neurons in the rostral ventral medulla studied with rat brainstem-spinal cord preparation. Auton Neurosci Basic Clin 2000; 84: 40-49.

10 Matsuura T, Kumagai H, Kawai A, Onimaru H, Imai M, Oshima N, Sakata K, Saruta T. Rostral ventrolateral medulla neurons of neonatal Wistar-Kyoto and spontaneously hypertensive rats. Hypertension 2002; 40: 560-565.

11 Matsuura T, Kumagai H, Onimaru H, Kawai A, ligaya K, Onami T, Sakata K, Oshima N, Sugaya T, Saruta T. Electrophysiological properties of rostral ventrolateral medulla neurons in angiotensin II la receptor knockout mice. Hypertension 2005; 46: 349-354.

12 Iigaya $\mathrm{K}$, Kumagai $\mathrm{H}$, Onimaru $\mathrm{H}$, Kawai A, Oshima N, Onami T, Takimoto C, Kamayachi T, Hayashi K, Saruta T, Itoh H. Novel axonal projection from the caudal end of the ventrolateral medulla to the intermediolateral cell column. Am J Physiol Regul Integr Comp Physiol 2007; 292: R927-R936.

13 Oshima N, Kumagai H, Onimaru H, Kawai A, Pilowsky PM, ligaya K, Takimoto C, Hayashi K, Saruta T, Itoh H. Monosynaptic excitatory connection from the rostral 
ventrolateral medulla to sympathetic preganglionic neurons revealed by simultaneous recordings. Hypertens Res 2008; 31: 1445-1454.

14 ligaya $K$, Kumagai $H$, Nabika T, Harada $Y$, Onimaru H, Oshima N, Takimoto $C$, Kamayachi T, Saruta T, Itoh H. Relation of blood pressure quantitative trait locus on rat chromosome 1 to hyperactivity of rostral ventrolateral medulla. Hypertension 2009; 53: 42-48.

15 Bourassa EA, Fang X, Li X, Sved AF, Speth RC. AT 1 angiotensin II receptor and novel non- $-\mathrm{AT}_{1}$, non- $\mathrm{AT}_{2}$ angiotensin II/III binding site in brainstem cardiovascular regulatory centers of the spontaneously hypertensive rat. Brain Res 2010; 1359: 98-106.

16 Chen D, Bassi JK, Walther T, Thomas WG, Allen AM. Expression of angiotensin type 1A receptors in $\mathrm{C} 1$ neurons restores the sympathoexcitation to angiotensin in the rostral ventrolateral medulla of angiotensin type $1 \mathrm{~A}$ knockout mice. Hypertension 2010; 56: $143-150$

17 Onimaru $\mathrm{H}$, Homma I. Whole cell recording from respiratory neurons in the medulla of brainstem-spinal cord preparations isolated from newborn rats. Pflugers Arch 1992; 420: 399-406.

18 Onimaru H, Homma I, Iwatsuki K. Excitation of inspiratory neurons by preinspiratory neurons in rat medulla in vitro. Brain Res Bull 1992; 29: 879-882.

19 Haas HL, Jefferys JG. Low-calcium field burst discharges of CA1 pyramidal neurones in rat hippocampal slices. J Physiol 1984; 354: 185-201.

20 Valenti VE, Ferreira C, Meneghini A, Ferreira M, Murad N, Ferreira Filho C, Correa JA, Abreu LC, Colombari E. Evaluation of baroreflex function in young spontaneously hypertensive rats. Arq Bras Cardiol 2009; 92: 205-215.
21 Cisternas JR, Valenti VE, Alves TB, Ferreira C, Petenusso M, Breda JR, Pires AC, Tassi $\mathrm{N}$, de Abreu LC. Cardiac baroreflex is already blunted in eight weeks old spontaneously hypertensive rats. Int Arch Med 2010; 3: 2.

22 Lundin S, Ricksten SE, Thorén P. Interaction between mental stress and baroreceptor control of heart rate and sympathetic activity in conscious spontaneously hypertensive (SHR) and normotensive (WKY) rats. J Hypertens Supp/ 1983; 1: 68-70.

23 Paton JF. The ventral medullary respiratory network of the mature mouse studied in a working heart-brainstem preparation. J Physiol 1996; 493: 819-831.

24 Mandel DA, Schreihofer AM. Modulation of the sympathetic response to acute hypoxia by the caudal ventrolateral medulla in rats. J Physiol 2009; 587: 461-475.

25 Sasaki S, Dampney RA. Tonic cardiovascular effects of angiotensin II in the ventrolateral medulla. Hypertension 1990; 15: 274-283.

26 Muratani H, Averill DB, Ferrario CM. Effect of angiotensin II in ventrolateral medulla of spontaneously hypertensive rats. Am J Physiol 1991; 260: R977-R984.

27 Alzamora AC, Santos RA, Campagnole-Santos MJ. Baroreflex modulation by angiotensins at the rat rostral and caudal ventrolateral medulla. Am J Physiol Regul Integr Comp Physiol 2006; 290: R1027-R1034.

$28 \mathrm{Li}$ YW, Guyenet PG. Angiotensin II decreases a resting $\mathrm{K}+$ conductance in rat bulbospinal neurons of the C1 area. Circ Res 1996; 78: 274-282.

29 Chapleau MW, Hajduczok G, Abboud FM. Pulsatile activation of baroreceptors causes central facilitation of baroreflex. Am J Physiol 1989; 256: H1735-H1741. 